\title{
The Effects of Gehuajiecheng Decoction on Expression of COX-2, Serum Level of PGE2, TNF- $\alpha$ and IL-6 in Alcoholic Hepatitis
}

\author{
Zhiwei Qu ${ }^{1, *}$, Wenbo Ju², Aihua Ren², Maoyang Liu ${ }^{3}$ \\ ${ }^{1}$ Department of Traditional Chinese Medicine, The Affiliated Hospital of Beihua University, Jilin City, China \\ ${ }^{2}$ Department of Anatomy, Beihua University, Jilin City, China \\ ${ }^{3}$ Department of Forensic Medicine, Beihua University, Jilin City, China
}

Email address:

zhiweiqu@sina.com.cn (Zhiwei Qu)

${ }^{*}$ Corresponding author

\section{To cite this article:}

Zhiwei Qu, Wenbo Ju, Aihua Ren, Maoyang Liu. The Effects of Gehuajiecheng Decoction on Expression of COX-2, Serum Level of PGE2, TNF- $\alpha$ and IL-6 in Alcoholic Hepatitis. International Journal of Chinese Medicine. Vol. 4, No. 2, 2020, pp. 21-26. doi: $10.11648 / \mathrm{j} . \mathrm{ijcm} .20200402 .12$

Received: April 22, 2020; Accepted: May 15, 2020; Published: May 29, 2020

\begin{abstract}
Background: In this experimental study, we have researched Gehuajiecheng decoction mechanism of action in the treatment of alcoholic hepatitis so as to supply an effective drug in clinical practice. Methods: 40 male Wistar rats were randomly divided into 4 groups and treated with different drugs. The control group were given intragastric administration of corn oil $(2 \mathrm{~g} / \mathrm{kg} / \mathrm{per}$ day $)$ and $50 \%$ glucose $(20 \mathrm{ml} / \mathrm{kg} / \mathrm{per}$ day); the model group were given intragastric administration of $40 \%$ alcohol $(8 \mathrm{~g} / \mathrm{kg} / \mathrm{per}$ day) and corn oil ( $2 \mathrm{~g} / \mathrm{kg} / \mathrm{per}$ day); Gehuajiecheng decoction group were given intragastric administration of Gehuajiecheng decoction $(13 \mathrm{~g} / \mathrm{kg} / \mathrm{per}$ day) and $40 \%$ alcohol $(8 \mathrm{~g} / \mathrm{kg} / \mathrm{per}$ day); the Celecoxib group were given intragastric administration of celecoxib (10mg/kg/per day) and $40 \%$ alcohol $(8 \mathrm{~g} / \mathrm{kg} / \mathrm{per}$ day). 30 days after treatment, the rats were anesthetized with sodium pentobarbital and the blood was collected. Serum levels of AST, ALT and GGT were measured by using chromatometry, PGE2, TNF- $\alpha$ and IL-6 levels were examined by using radioimmunology method. After taking blood samples, the rats were sacrificed, and the liver was determined by Western blotting analysis. Results: Compared to controls, model group showed significantly higher levels of serum AST, ALT, GGT, PGE2, TNF- $\alpha$ and IL-6, which can be corrected by administration of Gehuajiecheng decoction or celecoxib, since the group treated with Gehuajiecheng decoction or celecoxib showed significantly lower levels of serum AST, ALT, GGT, PGE2, TNF- $\alpha$ and IL-6 ( $<<0.01$ ). However, liver function and Inflammatory mediators in Gehuajiecheng decoction were not significantly lower than that of the celecoxib group ( $\mathrm{p}>0.05$ ). Similarly, COX-2 expression in the model group was significantly higher than that of controls. However, its expression can be depleted by Gehuajiecheng decoction or celecoxib as COX-2 expression in the model groups $(\mathrm{p}<0.01)$. Conclusion: Gehuajiecheng decoction protects against alcohol-induced liver injuries via inhibiting expression of COX-2, decreasing release of PGE2, TNF- $\alpha$ and IL-6. It has the same effect as COX-2 selective inhibitor, therefore, we suggest that this decoction could be used as an effective method in the treatment of alcoholic hepatitis.
\end{abstract}

Keywords: Gehuajiecheng Decoction, Alcoholic Hepatitis, Inflammatory Mediators, COX-2, Celecoxib

\section{Introduction}

Alcoholic hepatitis (AL) is one of the most common diseases in the world. Severe cases of alcoholic hepatitis can lead to liver injuries and dysfunctions, and further progresses to cirrhosis and liver cancer [1-4]. Although several medicines such as glucocorticoids, pentoxifylline, antioxidants, and
anti-TNF- $\alpha$ antibody have been used for clinical trial, currently, there is still no specific medicine for treatment of this disease [5-6]. From the perspective of traditional Chinese medicine, it provides a new way for the treatment of alcoholic hepatitis. Gehuajiecheng decoction was created by $\mathrm{Li}$ Dongyuan in the Jin and Yuan Dynasties. It is regarded as a special prescription for the treatment of alcoholic liver disease 
by doctors of all ages. Sometimes it is also called "Gehua Jiejiu Decoction", which comes from the theory of spleen and stomach. It is composed of 13 traditional Chinese medicines, such as Gehua, Fuling, Qingpi, Muxiang, Chenpi, Ginseng, Polyporus, fried Shenqu, Alisma, Ginger, Atractylodes macrocephala, cardamom and so on, which is one of the best antidotes to alcohol since ancient times, and also an effective antidote for alcoholic liver disease commonly used in modern times [7]. It was found in animal experiments that this prescription can reduce the blood alcohol content of experimental rats and has the effect of liver protection [8-12]. It has been reported that Gehuajiecheng decoction has the functions of protecting hepatocytes, anti fibrosis and reducing steatosis of hepatocytes [13-15]. We have discussed the experimental study of Gehuajiecheng decoction on alcoholic hepatitis.

\section{Materials}

Rabbit anti-mouse COX-2 polyclonal antibody was purchased from Cayman (USA). Mouse anti-rat $\beta$-actin monoclonal antibody was purchased from Sigma (USA). Biotin labeled goat anti-rabbit secondary antibody was purchased from KPL (UK). Goat anti-mouse/goat anti-rabbit secondary antibodies (Horseradish peroxidase conjugated) and chemiluminescence ECL were purchased from Beijing Zhongshan Biotechnology Co., Ltd (China). PGE2, TNF- $\alpha$ and IL-6 radioimmunology assay kits were purchased from Beijing North Institute of Biotechnology (China). The experimental prescription Gehuajiecheng decoction (Gehua $15 \mathrm{~g}$, nutmeg $15 \mathrm{~g}$, Amomum $15 \mathrm{~g}$, Muxiang $15 \mathrm{~g}$, Shenqu $15 \mathrm{~g}$, Ganjiang 6 g, Chenpi 6 g, Baizhu 6 g, Qingpi 6 g, Baifuling 6 g, Alisma 6 g, Polyporus 4.5g, Ginseng 4.5g, Licorice 9g) was purchased from Zhongjing pharmacy of Jilin City, and the decoction was prepared and concentrated to $135 \mathrm{ml}$ (the concentration of crude drug is $1 \mathrm{~g} / \mathrm{ml}$ ) for standby. Nitrocellulose membrane was purchased from Beijing Dingguo Biotechnology Co., Ltd (China). Celecoxib, a COX-2 selective inhibitor, was from Shuzhou pharmaceutical Co., Ltd (China). Other materials used in this study were purified.

\section{Method}

\subsection{Construction of Rat Models of Alcoholic Hepatitis}

40 male Wistar rats weighing 170-220g were purchased from the animal laboratory of Jilin University (License No: SCXK2003-007). The rats were randomly divided into 4 groups (model group, Gehuajiecheng decoction, celecoxib group, and control group, 10 of each group), and fed in separate cages (4-5 of each cage). All the rats except controls were given intragastric administration of alcohol $(8 \mathrm{~g} / \mathrm{kg} / \mathrm{per}$ day). The dose of alcohol was calculated using the following formula: dose of alcohol $(\mathrm{g})=$ the volume of alcohol $(\mathrm{ml}) \times$ the concentration of alcohol $(\mathrm{g} / \mathrm{ml}) \times 0.8$. The rats were regularly given intragastric administration of alcohol at $9 \mathrm{a}$. $\mathrm{m}$ and drug at
4 p. $m$ daily for 4 weeks. Animals in both the experimental and control groups were fed with ordinary granular feedingstuff and caged in a clean animal laboratory with temperature of $22 \pm 2^{\circ} \mathrm{C}$ and humidity of $70 \% \pm 5 \%$, eating and drinking freely. The control group $(\mathrm{n}=10)$ were given intragastric administration of corn oil (2g/kg/per day) and 50\% glucose $(20 \mathrm{ml} / \mathrm{kg} /$ per day); the model group $(\mathrm{n}=10)$ were given intragastric administration of $40 \%$ alcohol $(8 \mathrm{~g} / \mathrm{kg} / \mathrm{per}$ day) and corn oil ( $2 \mathrm{~g} / \mathrm{kg} / \mathrm{per}$ day); Gehuajiecheng decoction group (abbre. Gehua group) $(n=10)$ : Gehua group (13 g/kg/per day) and 40\% alcohol ( $8 \mathrm{~g} / \mathrm{kg} /$ per day) was given by gavage; the celecoxib group $(n=10)$ were given intragastric administration of celecoxib $(10 \mathrm{mg} / \mathrm{kg} / \mathrm{per}$ day) and $40 \%$ alcohol $(8 \mathrm{~g} / \mathrm{kg} /$ per day).

\subsection{Liver Blood and Tissue Preparations}

30 days after treatment, the rats were anesthetized with sodium pentobarbital. The abdominal aortic blood $(4-5 \mathrm{ml}$ of each rat) was collected under aseptic conditions and the rats were sacrificed. The blood was centrifuged at $4000 \mathrm{rpm}$ for 10 minutes and the supernatant was collected, aliquoted and stored at $-70^{\circ} \mathrm{C}$. The right lobe of liver was excised, washed in physiological saline, dried with filter paper, and fragmented into several pieces. One piece of liver tissues was fixed in $10 \%$ neutral buffered formalin, and the others were snap-frozen in liquid nitrogen and then kept at $-70^{\circ} \mathrm{C}$.

\subsection{Determination of Serum Levels of Various Indicators}

Serum levels of AST, ALT and GGT were measured by using Full Automatic Biochemical Analyzer (Hitachi, 7170, Japan). Serum levels of PGE2, TNF- $\alpha$ and IL-6 were examined by using commercial radioimmunology assay kits according to the manufacturer's manuals. Briefly, the samples were centrifuged at $3000 \mathrm{r} / \mathrm{min}$ for 3 seconds and stored at $-70^{\circ} \mathrm{C}$ until used. When required, the samples were centrifuged at $40^{\circ} \mathrm{C}, 3500 \mathrm{r} / \mathrm{min}$ for 15 minutes and the supernatants were used for measurement $(\mathrm{FJ}-2008 \mathrm{PS} \gamma$ counter).

\subsection{Histopathological Examination of Liver Tissue}

Following fixation with $10 \%$ neutral buffered formalin, the liver tissues were sliced, dehydrated with graded ethanol, stained with H\&E, and examined for histopathological morphology.

\subsection{Determination of COX-2 Expression in Liver Tissues}

Expression level of COX-2 in liver tissues was determined by Western blotting analysis. The fresh-frozen liver tissues were weighted, and lysed at ratio of 1: 10 in protein lysis buffer $(1 \%$ SDS, $1 \%$ triton $\mathrm{X}-100,1 \% \mathrm{NP}-40)$. The lysates were centrifuged twice at $10,000 \mathrm{rpm}$ for 5 minutes and the supernatants were collected. Protein concentrations were examined using Coomassie brilliant blue G250. A standard curve $(\mathrm{Y}=31.1402 \mathrm{X}+0.0318, \mathrm{Y}=$ protein concentration, $\mathrm{X}=\mathrm{OD}$ value) was generated and protein concentrations were then determined. A total of $150 \mu \mathrm{g}$ protein for each sample was subjected to discontinuous SDS-polyacrylamide gel 
electrophoresis (SDS-PAGE). Following electrophoresis, proteins were transferred onto nitrocellulose membrane in a semi-dry transfer buffer. The membrane was blocked in 5\% milk at room temperature for one hour and then incubated at $4^{\circ} \mathrm{C}$ overnight in primary antibodies. The following day, membranes were washed with TBS buffer and incubated in corresponding horseradish peroxidase-conjugated secondary antibodies at $37^{\circ} \mathrm{C}$ for 2 hours. Membranes were then washed in TBST, and protein bands were visualized by an Enhanced Chemiluminescence (ECL) and exposed to hyperfilm. The film was developed and the bands were analyzed using Bandscan software. The relative expression level of COX-2 was evaluated by $\mathrm{COX}-2 / \beta$-actin ratio.

\subsection{Statistical Analysis}

For comparing values obtained in different groups, one-factor analysis of variance (ANOVA) was performed by using SPSS22.0 software. $\mathrm{P}<0.05$ or 0.01 was considered to be statistically significant.

\section{Results}

\subsection{General Conditions}

No significant differences in weight of rats were observed between the control group and the other groups before and after treatment. During the treatment, all the rats in the control group were healthy and active, showing smooth coat, good spirit and appetite, and increased weight, whilst the rats in the model group suffered different degrees of drowsiness, showing dull coat and low spirit, reduced activities, loss of appetite and weight. The general conditions of rats in Gehua group and celecoxib group were slightly worse than those of controls but better than those of the model group.

\subsection{Pathological Changes of Liver Tissues}

Alcohol-induced liver injuries and its prevention by Gehuajiecheng decoction and celecoxib were confirmed by gross and microscopic examination of the liver in all the groups.

\subsection{Gross Observations}

The liver tissues of control rats were red-brown, and the surface was smooth and glistening. Whereas in the model group, the livers of rats were enlarged dramatically, had a pale yellow or yellow appearance, blunt edge and tight capsule, and adhered to surrounding tissues. Yellow-white focal degenerative lesions were visible, and the cut surface was greasy and cloudy. The pathological changes of liver of rats in Gehua group and celecoxib group were slightly severer than those of controls but milder than those of the model group. The color is darker than the control group but lighter than the model group. Yellow-white focal degenerative lesions were invisible, and the cut surface was still shiny and did not feel greasy.

\subsection{Light Microscopic Observations (H\&E Staining)}

In the control group, the structures of liver lobular were intact. Hepatocytes showed normal cell morphology and lined radiating outward from a central vein in the center (Figure 1). However, in the model group, the structures of liver lobular were in disorder. Mild ballooning degeneration of hepatocytes was widely observed, but necrosis of hepatocytes, infiltrating inflammatory cells and fatty liver changes had not been found (Figure 2). Gehua group (Figure 3) and celecoxib group (Figure 4) showed near intact structures of lobular, mild swollen hepatocytes, but no ballooning degeneration.

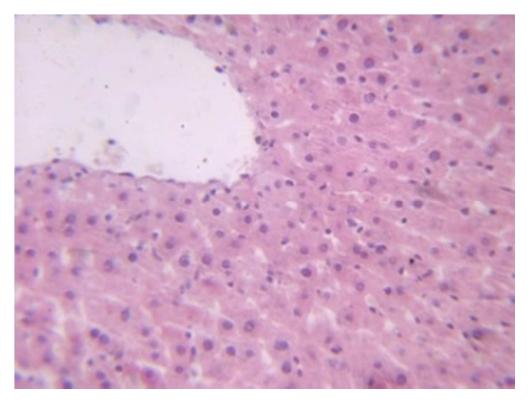

Figure 1. $\times 400$

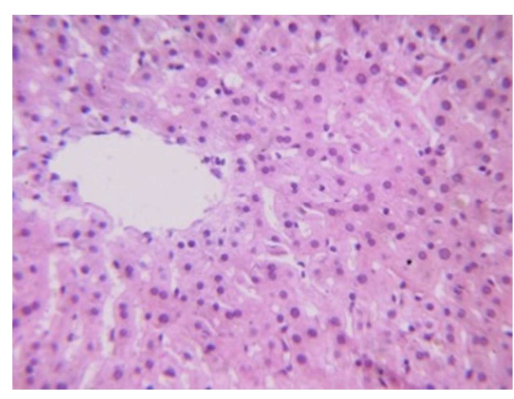

Figure 2. $\times 400$.

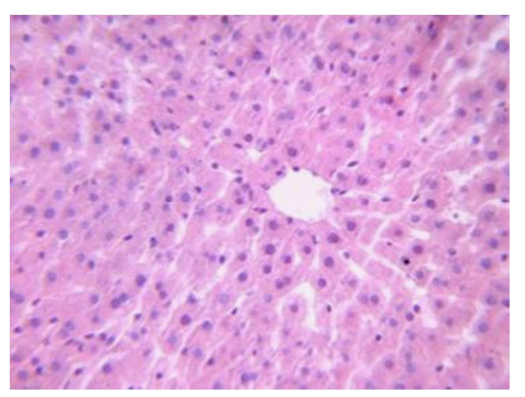

Figure 3. $\times 400$.

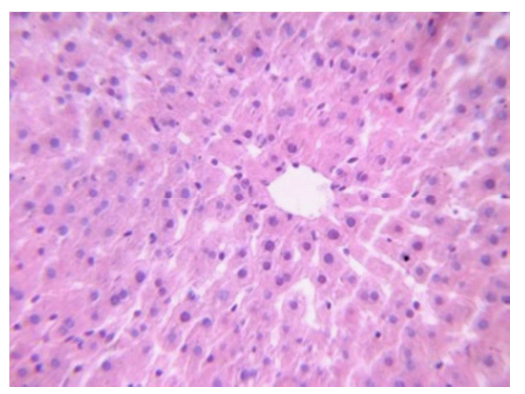

Figure 4. $\times 400$. 


\subsection{Serum Levels of AST, ALT and GGT}

Table 1. Comparisons of serum levels of AST, ALT and GGT in each group $(x \pm s)$.

\begin{tabular}{llll}
\hline group & n & serum ALT (IU/L) & serum AST (IU/L) \\
\hline Control group & 10 & $39.83 \pm 8.10$ & $52.7 \pm 13.35$ \\
Model group & 10 & $121.75 \pm 10.52^{*}$ & $146.05 \pm 12.39^{*}$ \\
Gehua group & 10 & $55.97 \pm 8.08^{\#}$ & $67.02 \pm 6.88^{\#}$ \\
Celecoxib group & 10 & $53.98 \pm 7.13^{\# \Delta}$ & $61.97 \pm 6.43^{\# \text { ム }}$ \\
$F$ ration & & 183.19 & 177.14 \\
\hline
\end{tabular}

*compared with control group $P<0.01,{ }^{*}$ compared with model group $P<0.01,{ }^{\star}$ compared with gehua group $\mathrm{P}>0.05$.

As shown in Table 1, serum levels of AST, ALT and GGT in the model group were significantly higher than those in the control group $(p<0.01)$. Serum levels of AST, ALT and GGT in either Gehua group or celecoxib group were significantly lower than those in the model group $(\mathrm{p}<0.01)$. No significant differences in serum ALT, AST and GGT were observed between Gehua group and celecoxib group ( $p>0.05)$. These results indicate that Gehuajiecheng might protect against alcohol-induced hepatotoxicity by decreasing serum levels of ALT, AST and GGT.

\subsection{Serum Levels of PGE2, TNF- $\alpha$ and IL-6}

Table 2. Comparisons of serum levels of PGE2, TNF- $\alpha$ and IL-6 ( $x \pm s)$.

\begin{tabular}{|c|c|c|c|c|}
\hline group & n & PGE2 (pg/ml) & TNF (pg/ml) & IL6 (pg/ml) \\
\hline Control group & 10 & $191.55 \pm 19.39$ & $0.85 \pm 0.09$ & $41.73 \pm 3.60$ \\
\hline Model group & 10 & $287.03 \pm 22.54 *$ & $1.88 \pm 0.12 *$ & $66.76 \pm 5.71^{*}$ \\
\hline Gehua group & 10 & $238.33 \pm 24.87^{\#}$ & $1.41^{`} \pm 0.16^{\#}$ & $58.96 \pm 5.00^{\#}$ \\
\hline $\begin{array}{l}\text { Celecoxib } \\
\text { group }\end{array}$ & 10 & $228.13 \pm 24.10 \#$ & $1.39 \pm 0.10^{\# \boldsymbol{\Lambda}}$ & $54.96 \pm 4.97^{\# \boldsymbol{\Lambda}}$ \\
\hline$F$ ration & & 29.74 & 124.16 & 45.99 \\
\hline
\end{tabular}

* compared with control group $P<0.01$; * compared with model group $P<0.05$; $\Delta$ compared with gehua group $\mathrm{P}>0.05$.

As shown in Table 2, serum levels of PGE2, TNF- $\alpha$ and IL-6 in model group were significantly higher than those in the control group $(p<0.01)$. Serum levels of PGE2, TNF- $\alpha$ and IL-6 in either Gehua group or celecoxib group were significantly lower than those in the model group $(\mathrm{p}<0.05)$. No significant differences in serum PGE2, TNF and IL-6 were observed between Gehua group and celecoxib group ( $\mathrm{p}>0.05)$. These results suggest that Gehuajiecheng decoction have effects of decreasing serum levels of PGE2, TNF- $\alpha$ and IL-6, and protecting hepatocytes from inflammatory damage.

\subsection{COX-2 Expression in Liver Tissues $(x \pm s)$}

To understand the impact of various treatments on liver COX-2 expression, the expression levels of COX-2 in liver tissues in all the four groups were examined by Western blotting analysis, and $\beta$-actin was used as a loading control (Figure 5). The relative expression levels of COX-2 were then presented and compared by the value of $\mathrm{COX}-2 / \beta$-actin ratio (Control group: $0.198 \pm 0.037, \mathrm{n}=10$; Model group: $0.422 \pm 0.050, \mathrm{n}=10$; Gehua group: $0.281 \pm 0.043, \mathrm{n}=10$; Celecoxib group: $0.252 \pm 0.060, \mathrm{n}=10)$. The control group showed low expression of COX-2, whilst the model group exhibited significantly higher expression level of COX-2 $(\mathrm{p}<0.01)$. Moreover, a significantly lower COX-2 expressions were found in either Gehua group or celecoxib group when compared to the model group $((\mathrm{p}<0.01)$.

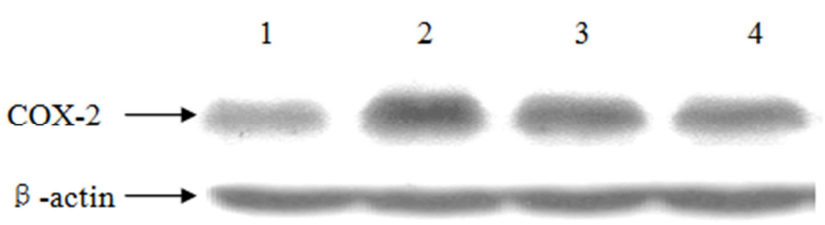

1. control group, 2. model group, 3. gehua group, 4. celecoxib group.

Figure 5. Expression of $C O X-2$ in the liver tissue.

\section{Discussion}

The main feature of acute alcoholic hepatitis is a series of inflammatory alterations, in company with liver injuries, the mechanisms of which include release of inflammatory mediators, peroxides, toxic oxygen, and Kupffer infiltrating. PGE2, TNF- $\alpha$, TGF and IL6 are considered to be critical inflammatory mediators involved in this process. PGE2 is a metabolic product of COX-2, while COX-2 is a subtype of $\mathrm{COX}$, a rate limiting enzyme that occurs during synthesis of PGs, COX-2 is not expressed or weakly expressed in normal tissues, but its expression level is markedly increased in the tissues with inflammation or tumors [16-18]. It has been previously shown that COX-2 expression is markedly increased in liver tissue of patients with alcoholic hepatitis. Elevated COX-2 expression increases PGE2 expression, aggravating liver injuries of alcoholic hepatitis [19]. In this study, we constructed the rat models of alcoholic liver injuries, and investigated the impact of Gehuajiecheng decoction on alcohol-induced liver injuries. We also examined COX-2 expression levels in liver tissues after various treatments, and explored the impact of Gehuajiecheng decoction and COX-2 selective inhibitor on alcoholic liver injuries. Our results demonstrated that COX-2 was weakly expressed in controls but highly expressed in the model group, The difference is significant. Compared to the model group, either Gehuajiecheng decoction or celecoxib group showed lower expression levels of COX-2, which is consistent with those reported by Bykov [19]. Bykov's study also revealed that serum levels of PGE2 and TNF $-\alpha$ elevated in alcoholic hepatitis, and a COX-2 inhibitor, celecoxib can dramatically reverse liver injuries [16]. Our results demonstrated that serum levels of PGE2, TNF- $\alpha$ and IL6 in the model group 
were significantly higher than that of controls. But after treatment of Gehuajiecheng decoction, serum levels of PGE2, TNF- $\alpha$ and IL6 significantly reduced compared to the model group. This suggests that Gehuajiecheng decoction decreases PGE2, TNF- $\alpha$ and IL6 levels and relieves inflammatory reactions.

Damage of mitochondria is usually the first presentation of alcoholic liver injuries. Since AST level in mitochondria of hepatocytes is much higher than that in hepatic sinusoid, serum AST will markedly elevate when the permeability of hepatocytes membrane increases although hepatocyte necrosis might not happen [19-22]. Therefore, serum AST is one of the sensitive indicators to estimate alcoholic liver injuries although it might not increase or just increase slightly in alcoholic hepatitis. In this study, although histological changes of hepatocytes did not reach the diagnostic standard of fatty liver or fibrosis, injuries were clearly observed. Thus, serum levels of ALT and AST in the model group were significantly higher than that of controls. The findings that serum levels of ALT and AST in Gehuajiecheng decoction or celecoxib groups were lower than those in liver injury group suggest that Gehuajiecheng decoction has the function of protecting alcohol-induced liver injuries.

GGT is a liver enzyme involved in the transport of amino acids and peptides into cells as well as glutathione metabolism. GGT is mainly found in membranes and microsome of hepatocytes and such as extremely sensitive to injuries of hepatocytes. When liver cells are injured, GGT is released to blood and elevated GGT level can be detected in serum [23]. The observation that serum level of GGT as well as AST and ALT in the model group was significantly higher than that of controls in this study is convincing evidence [24-27]. Serum GGT level in Gehua group or celecoxib group had a decrease, thus our results is that Gehua group reverses alcohol-induced liver cell injuries.

In conclusion, during the process of alcoholic liver injuries, inflammatory changes of liver in the early stage stimulate COX-2 expression and then induce the releases of PGE2, TNF- $\alpha$ and IL-6, which can be prevented by Gehuajiecheng decoction, resulting in relief of alcohol-induced liver injuries.

\section{Authors' Contributions}

Zhiwei $\mathrm{Qu}$ is responsible for experimental design and drug preparation, Wenbo $\mathrm{Ju}$ is responsible for histological and molecular biological experiments, Aihua Ren is responsible for data collection and statistical analysis, and Maoyang Liu is responsible for animal experiments, Dr. Qu is a major contributor in writing the manuscript, All authors read and approved the final manuscript.

\section{Abbreviations}

AL: Alcoholic hepatitis

COX-2: Cyclooxygenase 2

PGE2, ProstaglandinE2
TNF- $\alpha$ : Tumor Necrosis Factor- $\alpha$

IL-6: Interleukin 6

AST: Aspartate amino transferase

ALT: Alanine aminotransferase

GGT: Gamma-glutamyltransferase.

\section{Acknowledgements}

Professor Li Mingcheng from College of Medical Laboratory Science of Beihua University have contributed to the revision and finalization of later manuscripts, and Professor Dong Liping from department of statistics teaching have given great help to the data analyzed.

\section{References}

[1] Ashwani K Singal, Sudha Kodali, Lee A Vucovich, Victor Darley-Usmar, Thomas D Schiano Diagnosis and Treatment of Alcoholic Hepatitis: A Systematic Review. Alcohol Clin Exp Res. 2016; 40 (7): 1390-1402.

[2] Ling-Zu Kong, Nisansala Chandimali, Ying-Hao Han, Dong-Ho Lee, 4 Ji- Su Kim, 4 Sun-Uk Kim, Pathogenesis, Early Diagnosis, and Therapeutic Management of Alcoholic Liver Disease. Int J Mol Sci. 2019; 20 (11): 2712.

[3] Saggere Muralikrishna Shasthry, Shiv Kumar Sarin. New treatment options for alcoholic hepatitis. World J Gastroenterol. 2016; 22 (15): 3892-3906

[4] Fuster D, Samet JH. Alcohol Use in Patients with Chronic Liver Disease. N Engl J Med. 2018; 379 (13): 1251-1261.

[5] Sukhpreet Singh, Natalia A. O, Kusum K K. Treatment options for alcoholic and non-alcoholic fatty liver disease: A review. World J Gastroenterol. 2017; 23 (36): 6549-6570.

[6] S. Tome. Current management of alcoholic liver disease, Aliment Pharmacol Ther 2004; 19: 707-714.

[7] Li dongheng•jin, spleen and stomach, people's medical Press. China, 1st Ed., 2005: 81.

[8] Yao M, Liao Y, Li GQ, Law FC, Tang Y. Quantitative analysis of two isoflavones in Pueraria lobata flowers from eleven Chinese provinces using high performance liquid chromatography. Chin Med. 2010; 23 (5): 14.

[9] Zhang Z, Li S, Jiang J, Yu P, Liang J, Wang Y. Preventive effects of Flos Perariae (Gehua) water extract and its active ingredient puerarin in rodent alcoholism models, Chin Med. 2010; 5 (36): 2-8.

[10] Yang YY. Clinical observation of treatment of alcoholic fatty liver by Gehua Jiejiu Xiaozhi Decoction, Zhong Xi Yi Jie He Xue Bao. 2007; 5 (3): 343-5.

[11] Shi-li Jiang, Xu-dong Hu, Ping Liu. Immunomodulation and liver protection of Yinchenhao decoction against concanavalin A-induced chronic liver injury in mice, J Integ Med 2015; 13 (4): $262-267$.

[12] Feng R, Chen JH, Liu CH, Xia FB, Xiao Z, Zhang X, Wan JB. A combination of Pueraria lobata and Silybum marianum protects against alcoholic liver disease in mice, Phytomedicine. 2019; 58 (5): 152824. 
[13] Ding RB, Tian K, Huang LL, He CW, Jiang Y, Wang YT, Wan JB. Herbal medicines for the prevention of alcoholic liver disease: a review. J Ethnopharmacol. 2012; 144 (3): 457-65.

[14] Mou HY, Nie HM, Hu XY. Gutuo Jiejiu decoction improves survival of patients with severe alcoholic hepatitis: A retrospective cohort study. World J Gastroenterol. 2017; 23 (16): 2957-2963.

[15] Wang S, Yang FJ, Shang LC, Zhang YH, Zhou Y, Shi XL. Puerarin protects against high-fat high-sucrose diet-induced non-alcoholic fatty liver disease by modulating PARP-1/PI3K/AKT signaling pathway and facilitating mitochondrial homeostasis. Phytother Res. 2019; 33 (9): 2347-2359.

[16] Yun Shao, Kun Sun, Wei Xu, Xiao-Lin Li, Hong Shen, Wei-Hao Sun. Helicobacter pylori infection, gastrin and cyclooxygenase-2 in gastric carcinogenesis. World J Gastroenterol. 2014; 20 (36): 12860-12873.

[17] Raimundo F. d. A. J., Vinícius B. G., Renata F. d. C. L., Gerly A. d. C. B., Emilio d. C. M., Paulo M. M. Carvedilol Improves Inflammatory Response, Oxidative Stress and Fibrosis in the Alcohol-Induced Liver Injury in Rats by Regulating Kuppfer Cells and Hepatic Stellate Cells. PLoS One. 2016; 11 (2): e0148868.

[18] Jorge Castro-López, Antonio Ramis, Marta Planellas, Mariana Teles, Josep Pastor. Cyclooxygenase-2 immunoexpression in intestinal epithelium and lamina propria of cats with inflammatory bowel disease and low grade alimentary lymphoma. BMC Vet Res. 2018; 14: 158.

[19] Bykov L, Palmen M, Rainsford K. D, Lindros K. O. Chronic effects of celecoxib, a cyclooxygenase- 2 inhibitor, cause enhanced alcohol-induced liver steatosis in rats. Inflammopharmacology. 2006; 14: 36-41.
[20] Senthilkumar R, Nalini N., Effect of Glycineon tissue Fatty Acid Composition in an exprimental Model of Alcohol-Induced Hepatotoxity. Clin Exp Pharmacol Physiol. 2004, 31: 456-461.

[21] Saravanan N, Nalini N. Antioxidant effect of Hemidesmus indicus on ethanol-induced hepatotoxicity in rats. J Med Food. 2007 Dec; 10 (4): 675-82.

[22] Tipoe GL, Liong EC, Casey CA, Donohue TM Jr, Eagon PK, So $\mathrm{H}$, et al. A voluntary oral ethanol-feeding rat model associated with necroinflammatory liver injury. Alcohol Clin Exp Res. 2008; 32 (4): 669-82.

[23] Irie M, Suzuki N, Sohda T, Anan A, Iwata K, Takeyama Y, Watanabe H, Fischer P, Scherberich JE, Sakisaka S Hepatic expression of gamma-glutamyltranspeptidase in the human liver of patients with alcoholic liver disease. Hepatol Res. 2007; 37: 966-73.

[24] Elisabetta Ceni, Tommaso Mello, Andrea Galli. Pathogenesis of alcoholic liver disease: Role of oxidative metabolism World J Gastroenterol. 2014; 20 (47): 17756-17772.

[25] Arulkumar Nagappan, Dae Young Jung, Ji-Hyun Kim, Hoyoung Lee, Myeong Ho Jung. Gomisin N Alleviates Ethanol-Induced Liver Injury through Ameliorating Lipid Metabolism and Oxidative Stress. Int J Mol Sci. 2018; 19 (9): 2601.

[26] QQ. Dong, F Chu, CZ Wu, Q Huo, HY Gan, XM Li, H Liu. Scutellaria baicalensis Georgi extract protects against alcohol-induced acute liver injury in mice and affects the mechanism of ER stress. Mol Med Rep. 2016; 13 (4): 3052 3062 .

[27] Sougioultzis S, Dalakas E, Hayes PC, Plevris JN. Alcoholic hepatitis: from pathogenesis to treatment. Curr Med Res Opin 2005; 21 (9): 1337-46. 